\title{
1.1
}

\section{What is Glass?}

\section{An Introduction to the Physics and Chemistry of Silicate Glasses}

\author{
José-María Fernández-Navarro ${ }^{1}$ and María-Ángeles Villegas ${ }^{2}$ \\ ${ }^{1}$ Instituto de Óptica Daza de Valdés CFMAC, CSIC, Madrid, Spain \\ ${ }^{2}$ Instituto de Historia, CCHS, CSIC, Madrid, Spain
}

\subsubsection{Introduction}

The purpose of this introductory chapter is to present the basic concepts of the glassy state as well as the main properties of glasses that are different from those of other materials. There are two questions of theoretical interest to which many authors have paid special attention. These questions are the following: on the one hand, the establishment of structural models describing the behaviour of different kind of glasses and, on the other hand, the knowledge of the conditions that a substance must meet in order to obtain a glassy state (i.e. geometrical-structural factors, thermodynamics, kinetics, chemical bonding, etc., on which the ability to form a stable glass depends).

In this chapter only the main properties of glasses will be considered: viscosity and thermal expansion coefficient, affecting glasses during their heating and cooling, and the most important properties for their application and use, such as mechanical behaviour, optical transmission, reflectance and chemical durability.

The concept and meaning of the word glass depends on the corresponding context. In colloquial language the word glass is used to name objects made in this material (goblets, ophthalmic lenses, table vessels, etc.). In the scientific and technical world, the word glass is used for a wide series of materials with very different chemical composition, having all the fundamental physical and chemical characteristics that define the glassy state.

A material can be obtained in a glassy state through condensation from a gaseous phase, via cooling or polymerisation from a liquid phase or by disordering a solid phase. All of these pathways yield a noncrystalline structure. The most commonly used manner of obtaining a glass is the cooling of a liquid phase.

Modern Methods for Analysing Archaeological and Historical Glass, First Edition. Edited by Koen Janssens. (C) 2013 John Wiley \& Sons, Ltd. Published 2013 by John Wiley \& Sons, Ltd. 
Throughout history (and also nowadays) most glasses have been produced by the reaction of their components at high temperature, followed by melting and cooling of the resulting liquid phase under controlled conditions in order to avoid crystallisation.

\subsubsection{Fundamentals of the Glassy State}

\subsubsection{Transition Temperature}

When the cooling of a liquid is plotted versus the variation of a first-order parameter such as its specific volume, two different possibilities could occur (Figure 1.1.1). In the most common case, when the melting point is reached, both the liquid phase and the solid phase co-exist under equilibrium conditions and crystallisation suddenly takes place, accompanied by volume shrinkage. Below such temperature, cooling continues under equilibrium conditions and the volume of the solid phase diminishes according to its thermal expansion coefficient.

The other possibility is as follows: the temperature of the liquid drops below its melting temperature $\left(T_{f}\right)$ without any crystallisation. In this case a supercooled liquid is obtained, which is under metastable equilibrium inside the visco-plastic range. During the cooling, the viscosity significantly increases and progressively hinders the mobility of the atoms in the melt in such a way that they cannot order themselves in a crystalline symmetric sequence. Below the transition temperature $\left(T_{g}\right)$ the material reaches a thermodynamic non-equilibrium state and becomes rigid and brittle (glassy state). Thus, the transition temperature is the limit between the supercooled state and the glassy state. It is important to note that this transition temperature is not strictly constant, since it depends on the cooling rate. Independently of the glass composition, the $T_{g}$ value corresponds to a viscosity in the $10^{12.0}-10^{12.5} \mathrm{~Pa}$.s range. When cooling takes place quickly, the transition temperature slightly shifts towards higher values, and vice versa. If a glass is re-heated (annealed)

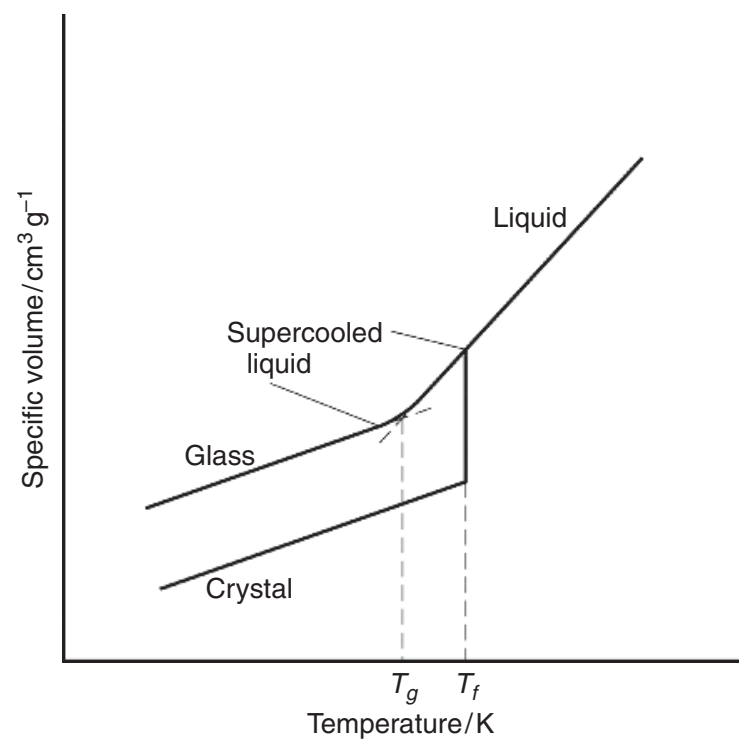

Figure 1.1.1 Variation of the specific volume of a glassy and a crystal phase of the same composition as a function of temperature. 
in its supercooling range $\left(T_{f}-T_{g}\right)$, the former process occurs reversibly if the heating rate is the same as the previous cooling rate.

The higher the difference between the melting temperature and the transition temperature (i.e. the wider the supercooling range $\left(T_{f}-T_{g}\right)$, the higher the thermal stability of the glass; in other words, the higher its tendency to form a glass.

\subsubsection{General Characteristics of Glasses}

Some of the main characteristics of glasses can be deduced from their behaviour during cooling. The most important is that glasses do not have a crystalline structure, due to the strong increase in viscosity that takes place during cooling, thereby hindering the regular ordering of the components. This implies that, from the thermodynamic point of view, glasses are frozen in an unstable state and therefore have an internal energy that is higher than that of the corresponding crystalline phase with the same composition. That is why glasses show structural relaxation phenomena under some thermal conditions, as well as reversible transition from the glassy state to the liquid state, without the appearance of crystalline phases.

\subsubsection{Definition of Glass}

When taking some of their properties into account, glasses have been defined in different ways. Many definitions are only based on a few characteristic aspects and, thus, are not satisfactory. Since glasses do not have an ordered structure, they can be considered as non-crystalline solids. However, from the point of view of their physical and chemical behaviour, glasses should be considered as supercooled liquids frozen in a rigid state. Both definitions are correct, but the first is more appropriate. Nevertheless, there are non-crystalline solids that are not glasses. The second definition is less adequate for those materials that show high mechanical rigidity and therefore behave as brittle solids.

In view of the most common industrial production process for glasses, the ASTM [1] standards define glass as an inorganic product of fusion which has cooled to a rigid condition without crystallization. This definition implies two restrictions. On the one hand, it limits the name glass to inorganic products and, on the other hand, to materials obtained by melting. However, there are many glasses of great technical interest that are organic materials, while other glasses can be obtained by special procedures (e.g. by the sol-gel method or by vapour deposition) different from melting. Therefore, neither the preparation procedure nor the chemical composition of glasses can be the main criterion to give a precise definition. As will be shown below, there are a wide variety of chemical families of glasses.

Due to the great chemical diversity of glasses, it is not possible to provide one general definition based on technical concepts concerning the practical applications of glasses and their particular properties. Conventional silicate glasses could be defined, with some limits, as inorganic non-crystalline products, mainly formed by silica, that are hard, brittle, generally transparent, with high chemical resistance and deformable at high temperature.

It appears more appropriate to define glasses from a physico-chemical point of view, according to their common behaviour, as materials that can be cooled below their supercooling range and reversibly heated above, without the appearance of crystalline phases [2].

\subsubsection{Criteria on the Formation of Glasses}

One question that arises when formulating a glass is to predict whether or not a (liquid) material with a specific composition can be frozen in a glassy state. Since this requires a strong increase in viscosity during cooling, it is useful to study the parameters that determine such a viscosity increase. This can be done on 
Table 1.1.1 Main criteria for glass formation.

\begin{tabular}{lll}
\hline Intrinsic characteristics & - Geometrical and structural (structural deforming, \\
& network cohesion) \\
& $\bullet \quad$ Chemical bond (bond nature and bonding energy) \\
Extrinsic characteristics & - Thermodynamic \\
\hline
\end{tabular}

the basis of different criteria (Table 1.1.1) that explain both the conditions for obtaining glassy materials (i.e. the glass-forming ability), and by means of numerical parameters that allow the classification of different substances according to their glass-forming ability.

\subsection{Geometric-Structural Criterion}

The first geometric criterion was stated by Goldschmidt [3], who proposed that a substance can be obtained as a glassy material when the ratio of its cation and anion radii lies within a particular range:

$$
R_{\text {cation }} / R_{\text {anion }}=0.2-0.4
$$

This ratio corresponds to tetrahedral coordination. Some years thereafter Zachariasen [4] enlarged this criterion and formulated the rules known as the Zachariasen rules: an oxide $\mathrm{A}_{\mathrm{m}} \mathrm{O}_{\mathrm{n}}$ can form a glass if the following conditions are fulfilled:

1. The number of anions to be coordinated with the central cation must be small ( 3 or 4$)$.

2. Each oxygen atom must be bonded with at least two A cations.

3. The coordination polyhedra must share vertices, never a side or an edge.

4. Each coordination polyhedron must share at least three vertices with other neighbouring polyhedra.

These conditions make possible the formation of a disordered structure that favours network deformability without too much stress on the bonds.

\subsection{Energy and Chemical-Bond-Based Criterion}

The ability of a substance to obtain a glassy state also depends on the bond strengths between their atoms. This parameter has been evaluated in a different way by several authors.

1.1.2.4.2.1 Theory of Field Intensity For Dietzel [5] the reference value is the field intensity of the cations in their respective oxides. This value is calculated as the $Z / a^{2}$ ratio, where $Z$ is the cation charge and $a$ is the distance between the cation and the oxygen atom; $a$ changes depending on the coordination. Cations with the highest field intensity are the best glass forming atoms, while those with low field intensity behave as glass modifying atoms.

1.1.2.4.2.2 The Single Bond Theory Sun [6] took the energy of the single bond $E_{\mathrm{A}-\mathrm{O}}$ as the reference for the glass-forming ability for an oxide $\mathrm{A}_{\mathrm{m}} \mathrm{O}_{\mathrm{n}} . E_{\mathrm{A}-\mathrm{O}}$ is defined as the ratio between the dissociation energy of the oxide $\mathrm{AO}_{\mathrm{n} / \mathrm{m}}$ in the gaseous state and the coordination number of the A cation. Similarly to the former 
theory, higher values of $E_{\mathrm{A}-\mathrm{O}}$ correspond to glass-forming oxides, while glass-modifying oxides have lower values. However, the obtained values do not fit with the experimental results for all cases and their relative differences are too small to form a representative scale of oxides.

1.1.2.4.2.3 The Thermal Energy Theory The anomalous behaviour of the previous theory was corrected by Rawson [7]. In addition to the bond energy, the thermal energy needed to break down such bonds should be taken into account. In this case, the indicator for glass-forming ability is the ratio of the single-bond energy to the oxide melting temperature expressed in degrees Kelvin $\left(E_{\mathrm{A}-\mathrm{O}} / T_{f}\right)$. Following this criterion, the best oxides to form a glass are those with a high $E_{\mathrm{A}-\mathrm{O}}$ and a low melting temperature. That is why glass formation is favoured for those compositions close to eutectic point areas.

1.1.2.4.2.4 The Mixed Bond Theory Smekal [8] considered the best glass-forming substances to be those in which mixed bonds (directed or not) co-exist, favouring the structural disorder needed for glass stability. According to this theory, substances that form glasses can be classified into three groups:

1. Inorganic compounds with ionic-covalent mixed bonds (e.g. oxide glasses)

2. Compounds with covalent-metallic bonds (e.g. selenide and telluride glasses)

3. Compounds with covalent-Van-der-Waals mixed bonds (e.g. organic glasses).

The co-existence of two kinds of bonds favours polymerisation, as well as molecular association in the melted state and, thus, freezing into the glass state.

1.1.2.4.2.5 The Electronegativity Theory $\quad$ On the basis of the electronegativity values stated by Pauling [9], Stanworth [10] calculated the covalence percentage of the chemical bond between two atoms on the basis of the difference of their electronegativity values. Bearing in mind the electronegativity of oxygen (3.5), the best glass-forming oxides are those with elements whose electronegativity is in the 1.7-2.0 range. That is, oxides that give rise to chemical bonds with electronegativity values between 1.8 and 1.5 , respectively. This corresponds to a covalence percentage in the $53-68 \%$ range, approximately.

\subsection{Thermodynamic Criterion}

The formation of a crystalline phase induces a decrease in the Gibbs free energy $\Delta G$ in the equation $\Delta G=\Delta H-T \Delta S$. This explains why it is only possible to obtain a glass from a melt when the change in free energy below the melting temperature $T_{f}$ is small, since this makes it difficult to form crystallization nuclei. At the melting temperature, the crystalline phase is in equilibrium with the liquid phase and $\Delta G=0$, so that

$$
\Delta S_{f}=\Delta H_{f} / T_{f}
$$

Thus, from the thermodynamic point of view, the vitrification of a melt is favoured in situations with a high melting entropy variation $\Delta S_{f}$. In other words, vitrification is favoured when there are important differences between the structural ordering of the melted phase and that of the crystalline phase. For this reason, the molar heat of melting $\Delta H_{f}$ should be high; this is the case in materials with a high bonding energy. For substances with similar heats of melting, those with lower melting temperatures can be easily obtained as glasses. This was foreseen by Rawson [7] in the theory explained in Section 1.1.2.4.2.3. 


\subsection{Kinetic Theory}

The formation of a glass from a melt is a kinetic process in which the cooling should take place at a sufficiently fast rate to inhibit the formation of crystallisation nuclei. Ultra-fast cooling procedures allow glasses to be obtained from substances where this is normally difficult. This is the case for some metals and alloys. Therefore, knowledge of the critical cooling rate to avoid formation of crystals is very important. Usually the volume of crystals $V_{x}$ in a glass is limited by the expression $V_{x} / V=10^{-6}$, where $V$ is the starting glass volume. This limit corresponds to the detection threshold of X-ray diffraction. Uhlmann and Yinnon [11] deduced that, for a given temperature, the volume of crystals formed during time $t$, can be calculated by the expression:

$$
V_{x} / V=1-\exp \left(-\pi / 3 v_{N} v_{C}^{4} t^{3}\right)
$$

where $v_{N}$ is the rate of nuclei formation and $v_{C}$ is the rate of crystals growth for such temperature. The knowledge of these values allows the representation of TTT curves (temperature, time and transformation). These curves indicate the time and the temperature combinations corresponding to a specific volume of crystals. Figure 1.1.2 shows typical TTT curves.

The curve vertex corresponds to the shortest time for crystallisation $\left(t_{m}\right)$; above this time formation of crystals starts for temperature $T_{m}$. TTT curves allow the calculation of the critical cooling rate at which the glass can be cooled without crystal formation. The critical cooling rate is approximately given by:

$$
\mathrm{d} T / \mathrm{d} t \approx\left(T_{f}-T_{m}\right) t_{m}
$$

where $T_{f}$ is the melting temperature.

\subsection{Summary}

From the previous paragraphs it can be deduced that the glass-formation phenomenon cannot be explained from a single point of view. This is due to the fact that process of obtaining glass is governed by different factors, some of which are closely related. Table 1.1.2 summarises the main factors favouring the formation of glasses.

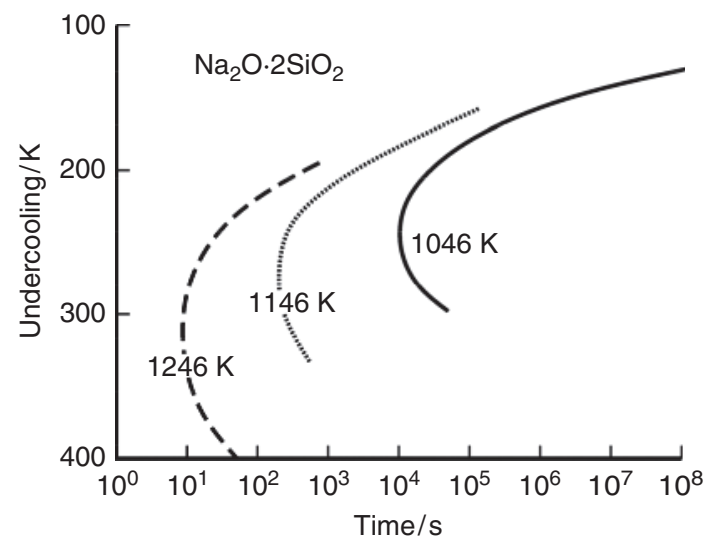

Figure 1.1.2 Time-temperature-transformation curves for $\mathrm{Na}_{2} \mathrm{O} .2 \mathrm{SiO}_{2}$-like materials having melting points of 1046, 1146 and $1246 \mathrm{~K} . V_{c} / V=10^{-6}$. After Uhlmann [11]. 
Table 1.1.2 Factors favouring glass formation

- Cations with low coordination number

- Cations enhancing structures with high network connectivity

- Cations with high field intensity

- Chemical bonds with high energy at melting temperature

- Mixed covalent chemical bonds with a covalent percentage of about 53-68\%.

- Large difference between entropy variation of crystalline phase and melted phase

- Low rate for crystalline growth

- Fast cooling

\subsubsection{Chemical Classification of Glasses}

There are a wide variety of substances of different chemical natures that can occur in the glassy state. Several glass families have appeared whose general names derive from the main component of their glassy network (Table 1.1.3). Within each glass family, it is possible to prepare glasses with very different composition. The components proportion can vary in very wide ranges, since glasses do not follow stoichiometric ratios.

The largest group is that of oxide glasses. Amongst oxide glasses the most important and most common, due to their applications throughout history, are silicate glasses, especially soda-lime silicate glasses. The following sections deal with this type of glasses.

\subsubsection{Silicate Glasses}

The most simple glass in this family is formed by $\mathrm{SiO}_{2}$ only. Its structure is a continuous network of [ $\left.\mathrm{SiO}_{4}\right]$ tetrahedra. The four vertices are shared by four neighbouring tetrahedra. Oxygen ions are the bonding bridges between tetrahedra and they ensure network continuity. The main structural difference between an $\mathrm{SiO}_{2}$

Table 1.1.3 Chemical classification of glasses.

\begin{tabular}{|c|c|c|}
\hline & Elements & $\begin{array}{c}\text { Chalcogens, metals } \\
\text { and alloys }\end{array}$ \\
\hline \multirow[t]{12}{*}{ Inorganic } & Oxides & Silicates \\
\hline & & Borates \\
\hline & & Phosphates \\
\hline & & Vanadates \\
\hline & & Germanates \\
\hline & & Tellurites \\
\hline & & Heavy metal oxides \\
\hline & Mixed oxides & Oxi-halides \\
\hline & & Oxi-nitrides \\
\hline & & Oxi-carbides \\
\hline & Non-oxides & Chalcogenides \\
\hline & & Halides \\
\hline Organic & \multicolumn{2}{|c|}{ Sugars, glycols, polymers, etc. } \\
\hline
\end{tabular}




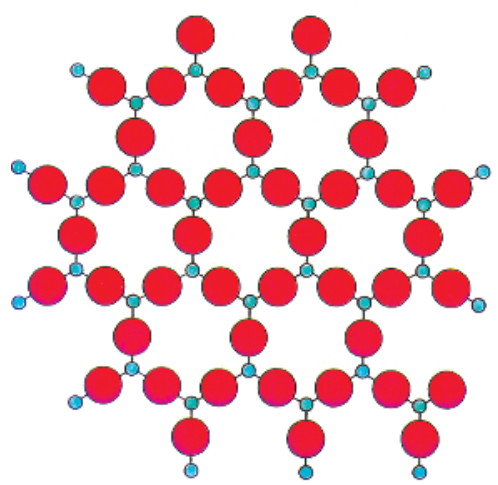

- Si

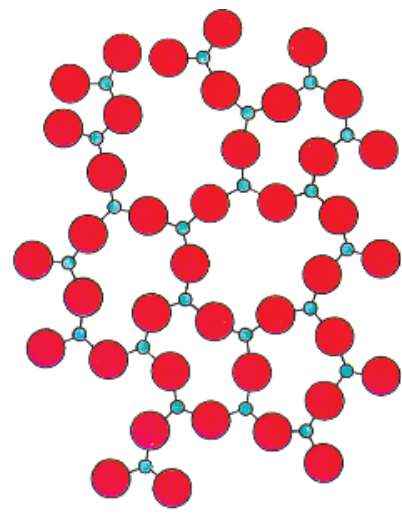

Figure 1.1.3 Planar plot from the structure of: (a) crystalline ordered $\mathrm{SiO}_{2}$ phase and (b) a random network of a pure $\mathrm{SiO}_{2}$ glass.

glass and an $\mathrm{SiO}_{2}$ crystal is the following: in the crystal the primary tetrahedral units are geometrically and periodically ordered in a regular network, while in the glass such units are randomly distributed, forming a distorted network (Figure 1.1.3), as Zachariasen [4] postulated. This was experimentally confirmed by Warren [12] using X-ray diffraction.

For pure silica glass, the coherent structure without network discontinuity points and the strength of the $\mathrm{Si}-\mathrm{O}$ bonds determine their low thermal expansion coefficient, high viscosity and high optical transmission range in the UV region. The incorporation of other oxides into the structure induces partial network opening. In the case of alkaline ions such as $\mathrm{Na}_{2} \mathrm{O}$, it occurs as follows:

$$
\equiv \mathrm{Si}-\mathrm{O}-\mathrm{Si} \equiv+\mathrm{Na}_{2} \mathrm{O} \rightarrow \equiv \mathrm{Si}-\mathrm{O}^{-} \mathrm{Na}^{+}+\mathrm{Na}^{+}{ }^{-} \mathrm{O}-\mathrm{Si} \equiv
$$

Each alkaline oxide molecule incorporated into the glass network forms two non-bridging oxygens, whose negative charge is neutralised by the positive charge of the alkaline ions (Figure 1.1.4). The alkaline ions are located in the network interstitial sites and are surrounded by oxygen ions. They modify both the structure and the properties of the glass and, thus, are named modifying ions. The incorporation of modifying ions induces increasing breakdown of siloxane bonds $\equiv \mathrm{Si}-\mathrm{O}-\mathrm{Si} \equiv$ and, therefore, a progressive weakness of the glass structure. Such weakness implies a decrease in both the softening temperature and viscosity of the glass, as well as an increase in both its thermal expansion coefficient and crystallisation tendency. As a general rule, the higher the network connectivity, the higher the glass stability. That is, the glass stability increases with the number of vertices of the coordination units shared with neighbouring polyhedra.

The formation of non-bridging oxygens, which are more polarisable than bridging oxygens, decreases the optical transmission in the UV range. Due to their intense ionic character, the $\mathrm{Na}^{+} \ldots{ }^{-} \mathrm{O}$ bonds are weaker than $\equiv \mathrm{Si}-\mathrm{O}^{-}$bonds. The $\mathrm{Na}^{+} \ldots{ }^{-} \mathrm{O}$ bonds become weaker as the field intensity of the corresponding alkaline ion decreases. That is why $\mathrm{K}^{+}$-ions, with a higher volume, but the same charge as $\mathrm{Na}^{+}$-ions, are weakly bonded to oxygen ions. Thus, they are more easily leached out than $\mathrm{Na}^{+}$-ions under chemical attack.

The incorporation of earth-alkaline oxides, for example $\mathrm{CaO}$, into the glass network takes place in a similar way to alkaline ones. However, due to their charge, each $\mathrm{Ca}^{2+}$-ion is bonded to two non-bridging oxygens and the breakdown of the $\equiv \mathrm{Si}-\mathrm{O}-\mathrm{Si} \equiv$ bonds is counteracted by the introduction of such divalent ions (Figure 1.1.4). 


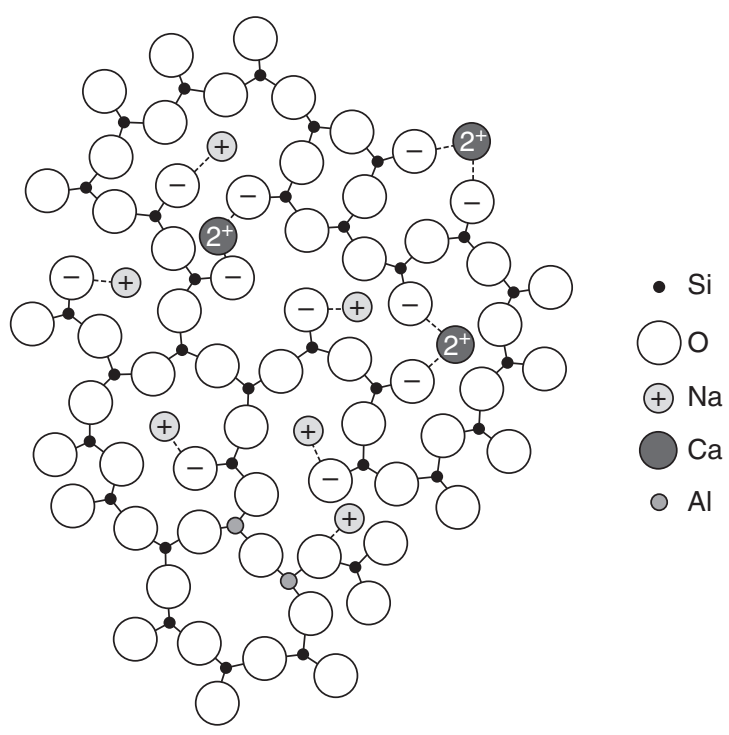

Figure 1.1.4 Planar plot from the structure of a soda-lime glass with a low content of $\mathrm{Al}_{2} \mathrm{O}_{3}$.

In conventional glasses the major components are $\mathrm{SiO}_{2}, \mathrm{Na}_{2} \mathrm{O}$ and $\mathrm{CaO}$. In addition, other components such as $\mathrm{K}_{2} \mathrm{O}, \mathrm{MgO}$ and $\mathrm{Al}_{2} \mathrm{O}_{3}$ are usually added to stabilise the glass and avoid devitrification. The aluminium oxide plays a very important role. When $\mathrm{Al}^{3+}$-ions are incorporated into the glass in a low proportion, they are coordinated as $\left[\mathrm{AlO}_{4}\right]$ tetrahedral groups. These units alternate with $\left[\mathrm{SiO}_{4}\right]$ groups in the glass structure and replace them in the role of network formers. However, since the oxygen/cation ratio in $\mathrm{Al}_{2} \mathrm{O}_{3}(3: 2)$ is smaller than in $\mathrm{SiO}_{2}(2: 1)$, the $\mathrm{Al}^{3+}$-ions, when surrounded by four oxygens have to incorporate one non-bridging oxygen to complete their coordination. These non-bridging oxygens become bridging oxygens and the structure connectivity increases. The negative charge excess in the $\left[\mathrm{AlO}_{4}\right]$ groups is compensated by the positive charge of the alkaline ions that are located closeby. When the molar concentration of $\mathrm{Al}_{2} \mathrm{O}_{3}$ is higher than those of the alkaline oxides $\left(\left[\mathrm{Al}_{2} \mathrm{O}_{3}\right] /\left[\mathrm{R}_{2} \mathrm{O}\right]\right)>1$, the number of non-bridging oxygens is too low

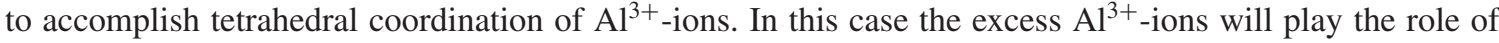
network modifiers and their coordination will be octahedral.

This structural model, based on a random network and without long-distance ordering, explains the isotropic behaviour of glasses. Moreover, the geometric irregularity of the distorted network induces an irregular distribution of the network energy, as a consequence of the different strengths in the chemical bonds. This is why glass does not have a defined melting point, but a variable wide thermal range of softening. Due to the infinite extension of the glass network, glasses possess a continuous structure without grain boundaries. This is an important difference from ceramic and metallic materials.

\subsubsection{Properties of Glasses}

\subsubsection{Glass Behaviour during Heating}

\subsection{Behaviour in the Rigid State: Thermal Expansion}

As with other materials, atoms in glasses increase their vibrations when the thermal energy rises due to heating. Thus, glasses experience an expansion process governed by a particular constant, the thermal 


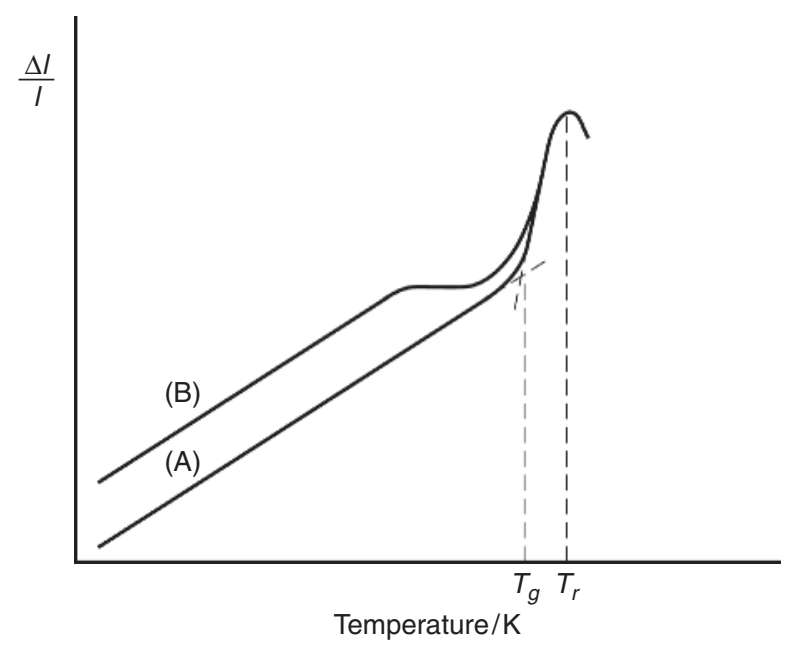

Figure 1.1.5 Thermal expansion curves of: (A) a well-annealed glass, and (B) a fast-cooled glass.

expansion coefficient, which can be defined as a length or volume variation per unit of temperature. In practise the average linear expansion coefficient $\alpha_{\Delta T}$ is usually employed:

$$
\alpha_{\Delta T}=1 / l_{0} \cdot \Delta l / \Delta T
$$

This coefficient is defined as the length variation $\Delta l$ experienced by a sample with initial length $l_{0}$ when heated over a temperature range $\Delta T$. The corresponding cubic expansion coefficient is $\beta \approx 3 \alpha$, approximately. In both cases the value of the expansion coefficient is expressed as a factor of $10^{-6}$ in $\mathrm{K}^{-1}$ units.

For temperatures below the transition temperature $T_{g}$, the thermal expansion coefficient of a correctly annealed glass is practically constant. Thus, the expansion curve of a slowly cooled glass (curve A in Figure 1.1.5), shows a linear variation up to the bottom limit of the annealing temperature $\left(T_{s}\right)$ (see Section 1.1.5.1.2.2). From this point the slope increases gradually up to the top limit of the annealing temperature. Above this point the expansion curve again shows linear behaviour that ends when the softening temperature $T_{r}$ is reached. At this stage apparent glass shrinkage, due to its plastic deformation, is observed.

In glasses suddenly cooled or annealed under inappropriate conditions, whose specific volume has been frozen at slightly higher dimensions than those of the equilibrium, the expansion curve has the appearance of curve B in Figure 1.1.5. During the first stage curves A and B are parallel, but before the bottom limit of the annealing temperature, a decrease in the slope occurs. This is due to a slight volume that becomes denser in the plastic relaxation range, in order to reach its equilibrium configuration.

Conventionally, from the technical point of view, hard glasses are those with expansion coefficient $\alpha<6.10^{-6} \mathrm{~K}^{-1}$, and soft glasses are those with $\alpha>6.10^{-6} \mathrm{~K}^{-1}$. Some expansion coefficients of several reference glasses are shown in Table 1.1.4.

Table 1.1.4 Average thermal linear expansion coefficients $\left[K^{-1}\right]$ for some reference glasses

\begin{tabular}{lccc}
\hline $\mathrm{SiO}_{2}$ & Sodium borosilicate & Soda-lime silicate & Lead crystal $(24$ wt. \% PbO) \\
\hline $0.5 \times 10^{-6}$ & $3.3 \times 10^{-6}$ & $8.7-9.0 \times 10^{-6}$ & $9.5-10.5 \times 10^{-6}$ \\
\hline
\end{tabular}


Table 1.1.5 Thermal shock resistance values for some reference glasses

\begin{tabular}{lcccc}
\hline Glass & $\alpha \times 10^{6}\left[\mathrm{~K}^{-1}\right]$ & $\mathrm{E}[\mathrm{GPa}]$ & $\mu$ & $\Delta T\left[{ }^{\circ} \mathrm{C}\right]$ \\
\hline Lead crystal & $9.5-10.5$ & $65-68$ & 0.23 & $\approx 58$ \\
Soda-lime & $8.7-9.0$ & $71-75$ & 0.22 & $\approx 65$ \\
Borosilicate & 3.3 & $64-65$ & 0.18 & $\approx 190$ \\
$\mathrm{SiO}_{2}$ & 0.5 & 72 & 0.16 & $\approx 1200$ \\
Eucryptite glass-ceramic & $\approx 0$ & $\approx 90$ & $\approx 0,20$ & $\approx \infty$ \\
\hline
\end{tabular}

The thermal expansion coefficient is one of the most important technical characteristics of glasses. On the one hand, it affects several aspects of glass production, for example the cooling rate during annealing, the generation of internal stresses and the ability to be tempered. On the other hand, from the technical application point of view, the thermal expansion coefficient plays an essential role in soldering glasses with other materials (ceramics, metals, glasses of different compositions), as well as in resistance against thermal shock. The soldering of both materials will be stable when the respective expansion coefficients are compatible, that is the difference between them should be $\Delta \alpha \leq 0.5 \times 10^{-6} \mathrm{~K}^{-1}$.

The glass resistance to fast temperature changes $\Delta T$ (thermal shock resistance) can be calculated from its mechanical strength $\sigma_{t}$, the Poisson number $(\mu \approx 0.15-0.25)$, and Young's modulus $E$. Furthermore $\Delta T$ depends on the form and thickness of the glass piece. For hollow glass objects, the valid formula is as follows:

$$
\Delta T=\sigma_{t}(1-\mu) / \alpha \mathrm{E}
$$

The thermal shock resistance mainly depends on the thermal expansion coefficient $\alpha$. Assuming a minimum real mechanical strength $\sigma_{t} \approx 50 \mathrm{MPa}$ for silicate glasses, the $\Delta T$ values given in Table 1.1.5 are obtained.

\subsection{Behaviour in the Plastic-Viscous State: Viscosity}

The plastic-viscous behaviour of glasses over a wide temperature range is one of their most important characteristics. As was explained in Section 1.1.2.1, the viscosity makes it possible that a melt could be frozen during cooling to a glassy state without crystallisation. Moreover, the viscosity plays an essential role throughout the stages of the industrial production of glasses (Figure 1.1.6). The efficiency of glass melting and refining processes are determined by the viscosity, as well as by the degree of homogeneity, the shaping process (by casting, blowing, pressing, drawing, etc.), the thermal devitrification ranges, and the degree of annealing and of stress relaxation.

The viscosity determines the sliding resistance produced between the molecules or structural aggregates in a fluid when it is under movement. The force needed to slide a horizontal fluid layer upon another is expressed by:

$$
F=\eta A \mathrm{~d} v / \mathrm{d} x
$$

where A is the contact surface area, $\mathrm{d} v / \mathrm{d} x$ is the perpendicular gradient of the flux rate throughout distance $x$, and $\eta$ is a constant, characteristic of each fluid, named the viscosity coefficient. Its value is expressed in Pa.s units, which replace poise $(\mathrm{P})$, the cgs system units used for decades. The equivalence between $\mathrm{Pa} \cdot \mathrm{s}$ and $\mathrm{P}$ is: $1 \mathrm{P}=1 \mathrm{dPa} \cdot \mathrm{s}=0.1 \mathrm{~Pa} . \mathrm{s}$. 


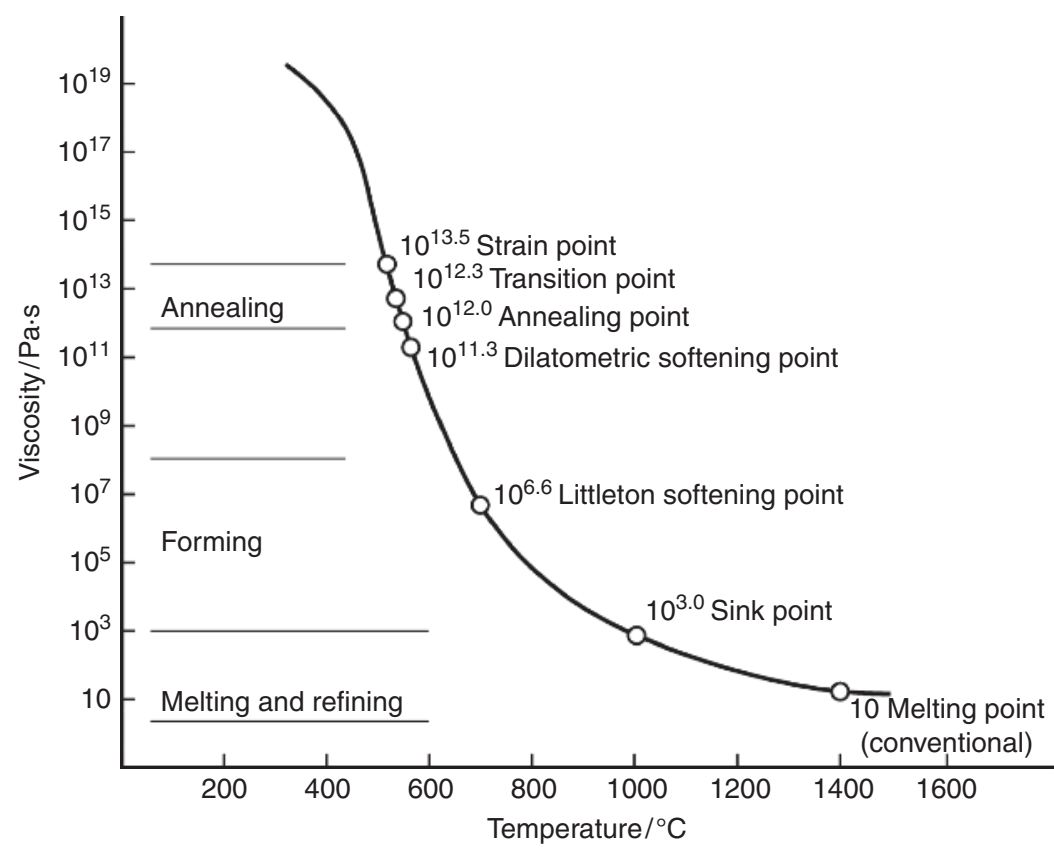

Figure 1.1.6 Characteristic viscosity-temperature curve of a soda-lime glass showing the most important viscosity fixed points and viscosity ranges for the different steps of a glass fabrication process.

1.1.4.1.2.1 Temperature Influence When a glass is heated above its transition temperature $T_{g}$, plastic-viscous behaviour occurs and the viscosity $\eta$ decreases exponentially according to the general expression:

$$
\eta=A \exp \left(E_{\eta} / R T\right)
$$

where $A$ is a constant, $R$ is the gas constant, $T$ the absolute temperature and $E_{\eta}$ the activation energy for viscous flow. The latter depends on the structural nature of each glass. Taking logarithms in the former equation, the following expression results:

$$
\log \eta=A^{\prime}+E_{\eta} / 19.15 T
$$

This indicates that, when $\log \eta$ is plotted as a function of $1 / T$, a straight line is obtained with a slope corresponding to the activation energy $E_{\eta}$. However, this only takes place when the fluid behaves as an ideal fluid, in which the molecular groups maintain their size and shape throughout the whole temperature range considered. Except for some glasses of very simple composition, for the majority of glasses a curve whose activation energy $E_{\eta}$ decreases with temperature is obtained.

In practice it is interesting to know the exact viscosity values over a wide range of temperatures; this includes $\eta$ values covering 18 orders of magnitude that span the most critical stages of the glass production process. The direct determination of viscosity throughout a more expanded range implies the use of different experimental methods, some of which are fairly complicated and time consuming. That is why several empirical formulas have been proposed that are only valid in limited temperature ranges. One of the best 
Table 1.1.6 Viscosity fixed points

\begin{tabular}{lll}
\hline Fixed points & \multicolumn{1}{c}{$\eta[\mathrm{Pa} \cdot \mathrm{s}]$} & Reference \\
\hline Strain point & $10^{13.5}$ & {$[16,17]$} \\
Transition point & $10^{12.0}-10^{12.5}$ & \\
Annealing point & $10^{12.0}$ & {$[16,17]$} \\
Dilatometric softening point & $10^{10.3}$ & \\
Littleton softening point & $10^{6.6}$ & {$[18,19]$} \\
Flow point & $10^{4.0}$ & {$[20]$} \\
Sink point & $10^{3.0}$ & {$[21]$} \\
Melting point (conventional) & 10 & \\
\hline
\end{tabular}

known and generally used is the Vogel-Fulcher-Tamman equation [13-15]:

$$
\log \eta=A+B /\left(T-T_{0}\right)
$$

This equation can be satisfactorily used in soda-lime silicate glasses over a wide temperature range. The application of this formula requires previous knowledge of the three constants $A, B$ and $T_{0}$. For this purpose the corresponding temperatures have to be experimentally measured at three fixed viscosity points. In general, the sink point, the Littleton point and the transition temperature $T_{g}$ are taken as references.

1.1.4.1.2.2 Viscosity Fixed Points Several viscosity fixed points have been defined for a wide scale of values. These points can be quickly and easily determined. The most important points are indicated in Table 1.1.6. Some of these points correspond to different stages in the glass production process (see Figure 1.1.6).

\subsubsection{Mechanical Behaviour}

\subsection{Elasticity Modulus}

Depending on the kind of deformation of materials submitted to mechanical stress, three main groups can be distinguished. When deformation remains after the stress stops, the material shows a plastic behaviour. When the material recovers its initial shape, it behaves as an ideal elastic material (e.g. glass behaviour). A third possibility is that a material exhibits an elastic deformation under a limited stress and a plastic deformation above a certain stress threshold (as is the case with metals).

When elastic materials are submitted to a traction stress, they follow Hooke's law:

$$
\sigma=E \varepsilon
$$

which assumes a direct proportion between the applied stress $\sigma$ and the produced deformation $\varepsilon$. The materialspecific proportionality coefficient $E$, is also called the elasticity modulus or Young's modulus. When the stress applied exceeds the material elasticity limit, the material breaks down without any residual deformation (brittle fracture). Glasses are characterised by brittle behaviour and a short elastic deformation range. The lower the $E$ value, the higher the elastic deformation experienced by the material. For a pure silica glass, Deeg [22] obtained an $E$ value of $720 \mathrm{kbar}(E=72 \mathrm{GPa})$. When modifier oxides are incorporated into the 
glass structure, the $\equiv \mathrm{Si}-\mathrm{O}-\mathrm{Si} \equiv$ bonds are broken and the network connectivity, as well as the $E$ value, progressively decreases. For the same reason, the $E$ modulus decreases when the glass temperature increases. This explains why $E$ also depends on the thermal history of the glass. Depending on the cooling conditions, Stong [23] measured $E$ values between 71 and $75 \mathrm{GPa}$ for conventional soda-lime silicate glasses.

\subsection{Theoretical Fracture Stress}

From the point of view of practical applications, knowledge of glass fracture stress is essential. Its theoretical value was calculated by Polanyi [24] from the energy $\gamma$ needed to form a new fracture surface and the interatomic equilibrium distance $a_{0}$ of the corresponding interatomic bonds. According to this, the theoretical fracture stress $\sigma_{t}$ needed to induce a breakdown is given by:

$$
\sigma_{t}=(4 \gamma E)^{1 / 2} / a_{0}
$$

Orowan [25] obtained a similar expression, whose difference with respect to Eq. 1.1.12 is that half the value for $\sigma_{t}$ is obtained.

For $\gamma \sim 10 \mathrm{~N} \mathrm{~m}^{-1}$, interatomic distances $a_{0}$ of $\sim 3.6 \times 10^{-10} \mathrm{~m}$ and an average $E$ of $\sim 70 \mathrm{GPa}$, the theoretical resistance values are very high, that is, between 10 and $30 \mathrm{GPa}$. However, the real fracture stress of common glasses is at about $5 \%$ below the theoretical value, that is, about 50 to $150 \mathrm{MPa}$. On the basis of the work of Inglis [26], Griffith [27] attributed this important difference to the presence of microcracks in the glass surface. Such microcracks could be formed by small stresses generated during glass cooling, due to compositional local microheterogeneities, or by minor damage caused by mechanical abrasion of the glass surface during use. These microcracks, not visible to the naked eye, have a semi-elliptic shape and penetrate some micrometers inside the glass (Figure 1.1.7), acting as multiplier centres for the applied stress $\sigma_{t}$. When a stress $\sigma$ is applied to the glass surface, it becomes concentrated in the crack vertex and from that point the fracture progresses. The value reached by the stress $\sigma_{r}$ in the microcrack extreme can be calculated as a function of the length $\lambda$ and the radius $r$ in the vertex, according to the expression:

$$
\sigma_{r}=2 \sigma(\lambda / r)^{1 / 2}
$$

For instance, for a fissure $5 \mu \mathrm{m}$ long with an extreme radius of $0.003 \mu \mathrm{m}$, the multiplier effect is approximately 80 times the applied stress.
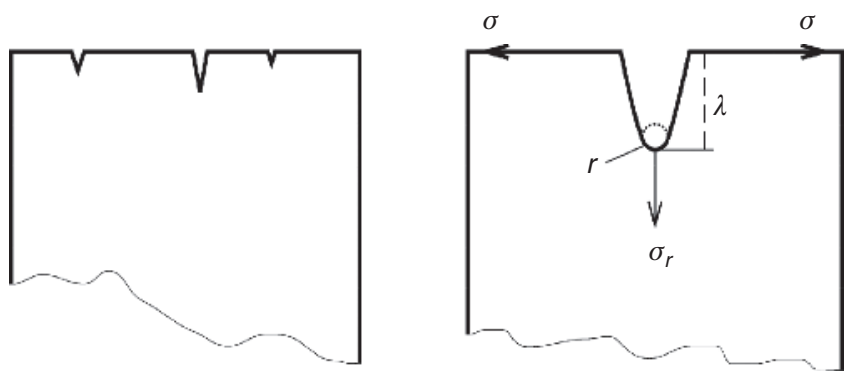

Figure 1.1.7 Ideal representation of microcracks at the glass surface. 


\subsection{Mechanism of Fracture Propagation}

Microcracks can grow to a critical size. When this critical size is reached, catastrophic fracture of the glass occurs. Crack growth involves the successive breakdown or opening of interatomic bonds. The energy required to do this can be supplied by mechanical stress, by aggressive action of a chemical medium or by the combined action of both. One of the chemicals favouring crack propagation is environmental moisture. Water is superficially adsorbed on surface sites with higher chemical reactivity, for example on microcrack extremes. By means of a dissociative chemisorption process, adsorbed water hydrolytically breaks down the siloxane bonds, upon which the water is incorporated into the glass structure, thereby forming $\mathrm{Si}-\mathrm{OH}$ groups (Figure 1.1.8). The $\mathrm{Si}-\mathrm{OH}$ formation causes the progressive opening of the network so that the microcrack penetrates deeper, while its extreme becomes more acute and its radius of curvature becomes smaller. Such changes favour fracture growth. This mechanism explains why glasses are better conserved under a dry atmosphere.

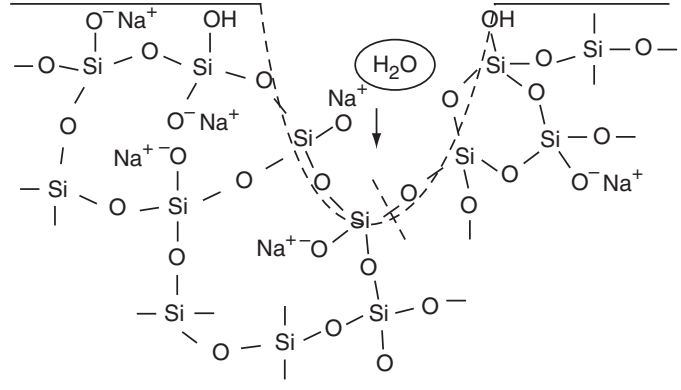

(a)

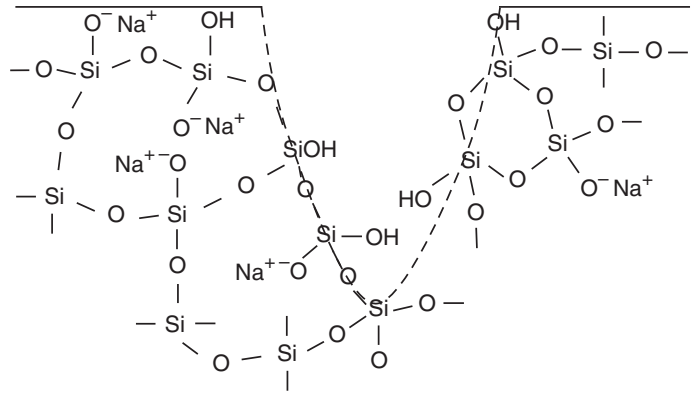

(b)

Figure 1.1.8 The chemical mechanism of the growth of glass microcracks.

\subsection{Toughness}

By means of fracture mechanics it is possible to understand better the mechanical behaviour of glasses, as well as their breakdown probability. The fracture mechanism works by intentionally introducing a crack into the material and calculating its resistance versus the crack propagation under different deformation conditions. One of the most important parameters to define mechanically brittle materials is the $K_{I C}$ or material toughness factor. This parameter represents the resistance of a solid against crack propagation [27]. There are three modes of fracture; amongst them the mode $I$ is the most frequently produced in brittle materials, in which the fracture surfaces are perpendicularly displaced. The toughness for this fracture mode is represented by $K_{I C}$. In order to determine the $K_{I C}$ factor, a method based on indentation measurements performed with a microdurometer has been developed. This device consists of a diamond indenter with a square pyramid shape (Vickers indenter), that induces square indentation prints. This method provides information on the Vickers microhardness $H_{V}$ and the shape of crack propagation in the material.

Indentation tests in glasses are carried out with a force of about $10 \mathrm{~N}$ and indentation times between 10 and $60 \mathrm{~s}$. From Vickers indentations, a value of $H_{V}$ is obtained as a function of the applied pressure $P$ and the half-diagonal $a$ of the indentation print formed.

$$
H_{V}=0.47\left(P / a^{2}\right) 9.8 \times 10^{-3}
$$


The indentation causes the appearance of cracks that start in the print vertices. $K_{I C}$ values can be calculated from the half-diagonal values $a$ and the crack length $c$, by using the following equations, developed by Lawn and Marshall [28]:

$$
\begin{aligned}
& K_{I C}=0.048(c / a)^{-1.32}\left(E / H_{V}\right)^{0.4} H_{V} a^{1 / 2} \quad \text { when } c / a \geq 2.5 \\
& K_{I C}=16\left(E / H_{V}\right)^{1 / 2}\left(P / c^{3 / 2}\right) \quad \text { when } c / a \leq 2.5
\end{aligned}
$$

The elasticity modulus $E$ can be calculated by means of the experimental values obtained by an indentation produced by a Knoop diamond indenter (rhombus-shaped imprint), using the expression:

$$
H_{V} / E=-2.222\left(b^{\prime} / a^{\prime}\right)+0.312
$$

where $a^{\prime}$ is the longer rhombus diagonal and $b^{\prime}$ the shorter one.

\subsubsection{Optical Behaviour}

The optical behaviour of conventional glasses is mainly governed by their refractive index, optical dispersion and absorbance. These are the parameters responsible for reflective power and brightness, the range of the chromatic spectrum during light refraction and the transparency at different wavelengths, respectively.

\subsection{Refractive Index and Optical Dispersion}

The refractive index $n$ is defined as the ratio between the velocity of light in vacuum $c_{0}$ and in the material $c_{m}$ :

$$
n=c_{0} / c_{m}
$$

Depending on the light wavelength, the refractive index varies: the higher the light wavelength, the lower the refractive index.

The refractive index for a glass results from the interaction of the incident light with the electrons of the glass atoms. As a consequence, polarisation or deformation of the orbitals takes place, the magnitude of which depends on a constant (named polarisability) characteristic for each material. This polarisation determines the light refraction, which is related to the dielectric constant $\varepsilon$ of the material. The molar refraction $R_{M}$ of a glass is given by the Lorenz-Lorentz formula:

$$
R_{M}=\left(n^{2}-1\right) /\left(n^{2}+2\right) \cdot M / \rho
$$

where $M$ is the molecular weight and $\rho$ the density.

For a glass the refractive index depends, on the one hand, on the polarisability of the glass ions and, on the other, on its molar volume $M / \rho$. The influence of anions is higher in comparison with cations, since anions are more polarisable. In oxide glasses the non-bridging oxygens in $\equiv \mathrm{Si}_{-}-\mathrm{O}^{-}$groups are more polarisable than bridging oxygens in siloxane $\equiv \mathrm{Si}-\mathrm{O}-\mathrm{Si} \equiv$ groups, which explains that pure silica glasses have a lower refractive index than soda-lime silicate glasses. While cations, with the electronic configuration of a noble gas, are not very polarisable, heavy oxide cations such as $\mathrm{Pb}^{2+}, \mathrm{Bi}^{3+}, \mathrm{La}^{3+}, \mathrm{Te}^{4+}, \mathrm{Ta}^{5+}$ and $\mathrm{W}^{6+}$, are much more polarisable, thus making a major contribution to the increase of the refractive index. 
The optical dispersion is defined as the variation of the refractive index with wavelength and is very useful for optical glasses. The optical dispersion is measured by the difference between the refractive indexes at two wavelengths.

\subsection{Reflectance and Brightness}

The brightness of an object depends on the reflectance $R$ of the material it is made of; $R$ is given by the ratio between the reflected light intensity $I_{R}$ and the incident light intensity $I_{0}$. When a light ray penetrates perpendicularly through a medium with refractive index $n=1$ (e.g. the air with $n_{\mathrm{ai}}=1.0003$ ), on a plate glass surface with refractive index $n$, the reflected light intensity on each glass side is:

$$
R=(n-1)^{2} /(n+1)^{2}
$$

Therefore, for a simple glass sheet with two sides, the light intensity that passes through is:

$$
I=I_{0}\left(1-R^{2}\right)
$$

In conventional soda-lime silicate glasses with refractive index $n=1.5$, the reflection on each side is 0.04 , that is, $4 \%$ intensity is lost upon crossing the interface between the two media (glass and air). Therefore, the light intensity that passes through the glass is $92.16 \%$ of the incident light. The higher the refractive index of a glass, the higher its reflectance and brightness, and the lower the percentage of transmitted light.

\subsection{Optical Transmission}

One of the main characteristics of glasses is transparency to visible light. This property is relevant in most important applications of glasses. In the same way as other dielectric materials, glasses are transparent because their strongly bonded electrons are not excitable by the visible light energy. Thus, they do not interact with the light photons.

The spectral transmission range for a conventional soda-lime silicate glass without colouring ions is between $\lambda=380 \mathrm{~nm}$ (corresponding to the near ultraviolet region) and $\lambda=2500-3000 \mathrm{~nm}$ (in the near infrared region) (Figure 1.1.9). Glasses are not transparent to ultraviolet light because this radiation, with a higher energy than that of visible light, excites the electrons of the oxygen bonds and therefore becomes absorbed. When the number of non-bridging oxygens increases, the network bonds are weaker and the ultraviolet absorption edge shifts toward a higher wavelength. Conversely, pure silica glasses, which only have bridging oxygens, have a higher transparency in the ultraviolet range. In fact, their absorption edge can reach up to $170 \mathrm{~nm}$.

In the infrared (IR) region, the absorption of radiation takes place when its frequency is in resonance with those of interatomic vibrations. The transmission range of glass in the IR can be enlarged by incorporating heavy metal oxides (HMO).

If a glass contains elements that selectively absorb radiation of specific visible-light wavelengths, it will show a colour due to the chromatic additive result of all the other non-absorbed spectral components. The transmitted colour intensity is given by the Lambert-Beer law:

$$
I=I_{0} \exp (-\varepsilon c d)
$$

where $I$ and $I_{0}$ are the transmitted and incident light intensities, respectively, $\varepsilon$ is the molar absorption coefficient or molar absorptivity, $c$ is the molar concentration (in $\mathrm{mol}^{-1}$ ) of the absorbent substance and $d$ is 


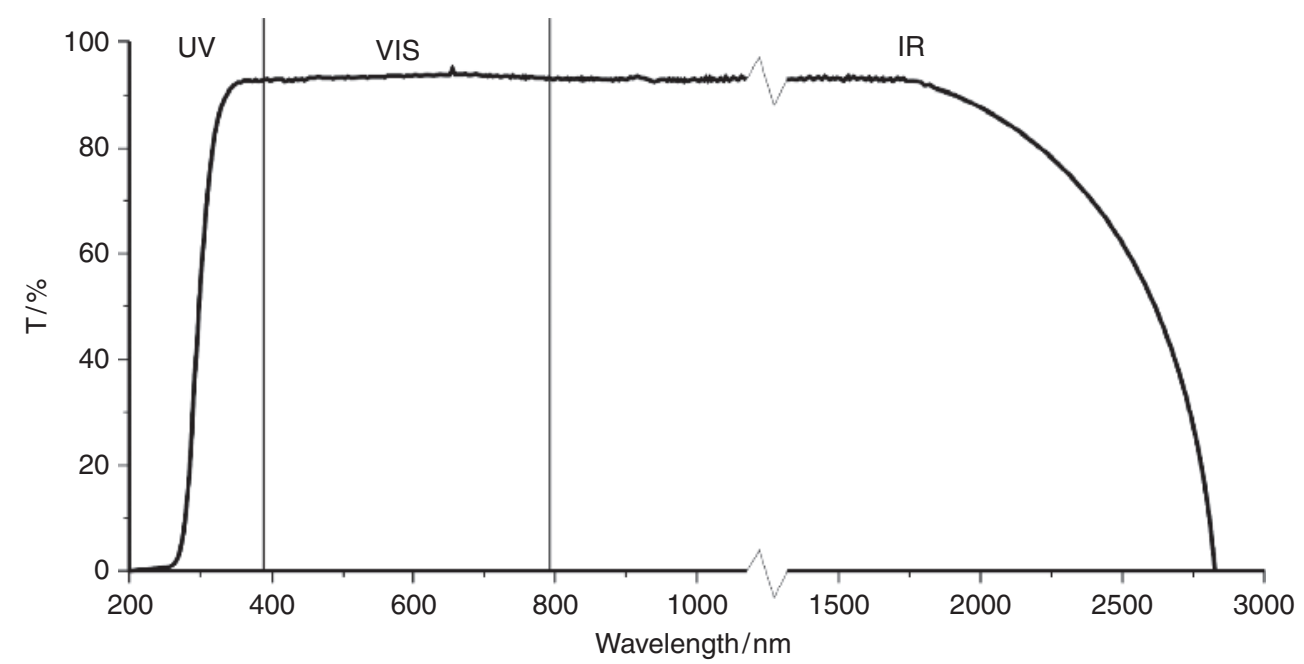

Figure 1.1.9 Optical absorption spectrum from a conventional colourless soda-lime glass.

the sample thickness (in $\mathrm{cm}$ ). The different colouring substances for glasses are described in another chapter of this book.

\subsubsection{Chemical Behaviour}

Besides transparency, the main property of silicate glasses that has made them very useful for many applications throughout history is their high chemical durability, which is particularly high for pure silica glasses. In contrast, silicate glasses containing alkaline ions are prone to attack by aqueous media. The corresponding reactions proceed via different mechanisms depending on the $\mathrm{pH}$ (acid, neutral or basic) of the medium.

\subsection{Attack in Acidic Media}

Acid attack of the glass begins at the surface by an ion exchange between the $\mathrm{H}^{+}$-ions of the medium with the alkaline ions of the glass. For instance:

$$
\mathrm{Na}^{+}{ }^{-} \mathrm{O}-\underset{\mid}{\mathrm{Si}}-\mathrm{O}-\underset{\mid}{\mathrm{Si}}-\mathrm{O}^{-} \mathrm{Na}^{+}+\mathrm{H}_{2} \mathrm{SO}_{4} \rightarrow \mathrm{HO}-\underset{\mid}{\mathrm{Si}}-\mathrm{O}-\underset{\mid}{\mathrm{Si}}-\mathrm{OH}+\mathrm{Na}_{2} \mathrm{SO}_{4}
$$

This reaction causes a progressive dealkalinisation of the glass that proceeds very slowly. Alkaline ions with higher volumes are weakly bonded to the non-bridging oxygens, due to their lower field intensity, and they are easily leached from the glass network. This explains why potash glasses can be attacked more easily than soda glasses. Earth-alkaline ions are firmly bonded to the network by means of their double charge and they are only leached when the glassy network has seriously deteriorated. In general, soda-lime silicate glasses show good chemical durability against most acids. However, silicate glasses are strongly attacked by hydrofluoric acid (HF) which destroys the glass network by dissolving their components. In this process, fluoride ions substitute the oxygen ions and directly bond to silicon atoms to form the volatile $\mathrm{SiF}_{4}$, as well as 
alkaline and earth-alkaline fluorosilicates. To a lesser extent, soda-lime silicate glasses can be also attacked by some phosphoric acids.

\subsection{Attack in Basic Media}

In basic media the chemical attack on glasses is the most dangerous, since $\mathrm{OH}^{-}$groups break down siloxane $\equiv \mathrm{Si}-\mathrm{O}-\mathrm{Si} \equiv$ bonds, following the reaction:

$$
\equiv \mathrm{Si}-\mathrm{O}-\mathrm{Si} \equiv+\mathrm{OH}^{-} \rightarrow=\mathrm{Si}-\mathrm{OH}+{ }^{-} \mathrm{O}-\mathrm{Si} \equiv
$$

In the presence of water, new $\mathrm{OH}^{-}$groups are formed:

$$
\equiv \mathrm{Si}-\mathrm{O}^{-}+\mathrm{H}_{2} \mathrm{O} \rightarrow=\mathrm{Si}-\mathrm{OH}+\mathrm{OH}^{-}
$$

As this reaction proceeds, more and more bridging oxygen bonds are broken down and the glassy network is progressively destroyed.

\subsection{Attack in Neutral Aqueous Media}

In neutral aqueous media, slight glass dealkalinisation takes place, due to exchange of $\mathrm{H}^{+}$-ions with alkaline ions from the glass:

$$
\equiv \mathrm{Si}-\mathrm{O}^{-} \mathrm{Na}^{+}+\mathrm{H}^{+} \mathrm{OH}^{-} \rightarrow \equiv \mathrm{Si}-\mathrm{OH}+\mathrm{Na}^{+} \mathrm{OH}^{-}
$$

This reaction is not dangerous if the alkaline hydroxide formed is removed or diluted to a sufficient extent. However, when a small amount of water remains in contact with the glass surface for a long time, under a wet saturated atmosphere that prevents vaporisation, the alkaline ions concentration increases, yielding a strongly aggressive basic medium that will favour the mechanism described in Section 1.1.4.4.2. This is the case of the very small water droplets formed by condensation in a stationary wet atmosphere.

\subsection{The Influence of Several Factors on the Chemical Durability of Glass}

As can be deduced from the previous section, one of the factors determining the mechanism of chemical attack on glasses that strongly affects its speed of progress is the basicity of the medium. The higher the $\mathrm{pH}$ value, the more aggressive the attack reaction that progressively breaks down bridging oxygen bonds and thus destroys the glassy network. Even pure silica glass is attacked by strongly alkaline media, as El-Shamy et al. [29] demonstrated by means of tests using solutions with increased $\mathrm{pH}$ values (Figure 1.1.10). While the glass remains almost unaltered under acid or slightly basic media, the higher the medium basicity above $\mathrm{pH}$ 8, the higher the amount of $\mathrm{SiO}_{2}$ leached.

Besides the influence of the $\mathrm{pH}$ on the chemical durability of glasses, other important factors have to be considered, for example, temperature, attack time and glass composition.

As far as temperature is concerned, the degree of attack $D$ varies following the Arrhenius equation:

$$
D=A \exp (-E / R T)
$$

where $A$ is a constant, $R$ is the gas constant and $E$ is the activation energy. The $E$ value varies between 60 and $80 \mathrm{~kJ} \mathrm{~mol}^{-1}$, depending on the glass composition. The influence of the attack time depends on the kind 


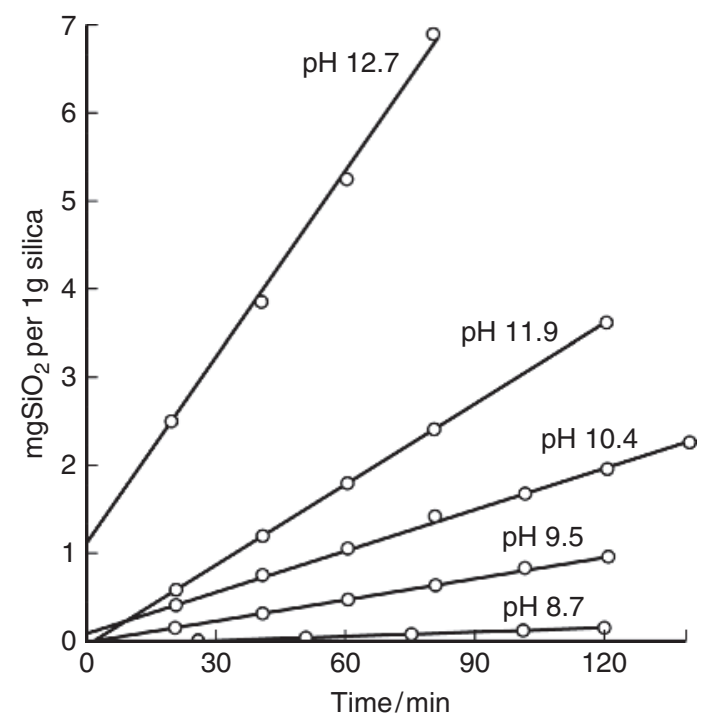

Figure 1.1.10 Time dependence of the removal of silica from fused silica powder for various $\mathrm{pH}$ values at $80^{\circ}$ C. After El-Shamy, Lewins and Douglas [29].

of chemical process involved. When it proceeds through ion-exchange between glass alkaline ions and the $\mathrm{H}^{+}$-ions of the aqueous medium, the process fits a pure diffusion mechanism. In this case the kinetics vary proportionally with the square root of time $\left(t^{1 / 2}\right)$, as Rana and Douglas [30] demonstrated (Figure 1.1.11). At high temperature the attack is more aggressive and, in basic media this induces destruction of the glassy network that is proportional to time (Figure 1.1.10).

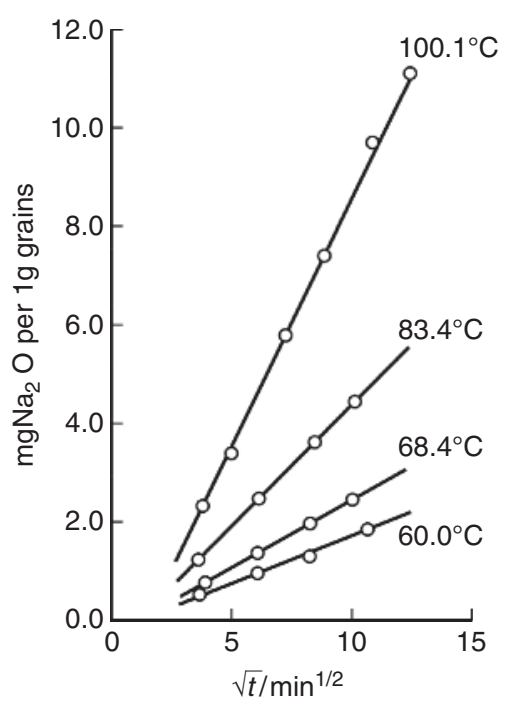

Figure 1.1.11 $\mathrm{Na}_{2} \mathrm{O}$ miligrams extracted from a $20 \mathrm{Na}_{2} \mathrm{O} .80 \mathrm{SiO}_{2}$ glass in pure water at different temperatures after short leaching time as a function of the square root of time. After Rana and Douglas [30]. 
From the point of view of chemical composition, binary alkaline silicate glasses $\left(\mathrm{R}_{2} \mathrm{O}_{\mathrm{SiO}} \mathrm{SiO}_{2}\right)$ offer the lowest resistance to chemical attack. Under basic or neutral media the amount of alkaline ions leached increases from lithium to potassium, that is, following the decreasing value of the cation field intensity. Within the series $\mathrm{Li}^{+}-\mathrm{K}^{+}$, the $\mathrm{SiO}^{-} \mathrm{R}^{+}$bond strength decreases as well, so that the glass becomes more easily attackable. In ternary glasses earth-alkaline oxides incorporation reinforces the glassy network which, in turn, reduces the probability of chemical attack.

The incorporation of some network formers is of clear benefit by increasing resistance to basic media. For instance, $\mathrm{B}_{2} \mathrm{O}_{3}$ (borosilicate glasses such as Pyrex ${ }^{\circledR}$ and $\operatorname{Duran}^{\mathrm{B}}$ ), $\mathrm{Al}_{2} \mathrm{O}_{3}$ and, above all, $\mathrm{ZrO}_{2}$, which allows the development of glass fibres with high alkaline resistance used to reinforce cement panels $\left(\mathrm{Cemfil}^{\mathbb{B}}\right.$ glasses), improve the durability of glass.

\subsection{Surface Alteration of Glasses}

In aqueous media, simultaneously to the attack reactions that take place, water molecules are incorporated into the surface, forming a thin gel layer. Scholze et al. [31] calculated that 0.46 water molecules enter per $\equiv \mathrm{Si}-\mathrm{OH}$ group formed. The gel layer forms a superficial interface that delays or prevents further chemical attack inside the glass body. Nevertheless, when a wet environment is retained for too long, for example, in glasses buried for many years, dealkalinisation due to entry of the water molecules causes local variations in volume near the surface. Thus, stresses causing cracks and detachments are generated that, in their turn, induce the formation of thin stratified layers. These layers are responsible for light interference phenomena that produce the well-known iridescence effect on the surface of degraded glass objects.

\section{References}

1. American Standard Testing Materials. (1997) Standard terminology of glass products. ASTM C 162-97. West Conshohocken, USA.

2. Fernández Navarro, J.M. (2003) El vidrio, Consejo Superior de Investigaciones Científicas, Madrid.

3. Goldschmidt, V.M. (1929) Crystal structure and chemical composition. Trans. Faraday Society, 25, $253-283$.

4. Zachariasen, W.H. (1932) The atomic arrangement in glass. J. Am. Ceram. Soc., 54, 3841-3851.

5. Dietzel, A. (1942) Die Kationenfeldstärken und ihre Beziehungen zu Entglasungsvorgängen, zu Verbindungsbildung und zu den Schmelzpunkten von Silikaten. Z. Elektrochem., 48, 9-23.

6. Sun, K.H. (1947) Fundamental condition of glass formation. J. Am. Ceram. Soc., 30, 277-281.

7. Rawson, H. (1956) The relationship between liquidus temperature, bond strength and glass formation. IV Congrès International du Verre, Paris, pp. 62-69.

8. Smekal, A. (1949) Über die Natur der glasbildenden Stoffe. Glastech. Ber., 22, 278-289.

9. Pauling, L. (1948) The Nature of the Chemical Bond, Cornell University Press, New York.

10. Stanworth, J.E. (1948) The ionic structure of glass. J. Soc. Glass Technol., 32, 366-372.

11. Uhlmann, R.D. and Yinnon, H. (1983) The Formation of Glasses, in Glass Science and Technology, Vol. 1, Glass Forming Systems (eds R.D. Uhlmann and N.J. Kreidl), Academic Press, New York-London, pp. 1-47.

12. Warren, B.E. (1941) Summary of work on atomic arrangement in glass. J. Am. Ceram. Soc., 24, 256-261.

13. Vogel, H. (1921) Das Temperaturabhängigkeitsgesetz der Viskosität von Flüssigkeiten. Phys. Z., 22, $645-646$.

14. Fulcher, G.S. (1925) Analysis of recent measurements of the viscosity of glasses. J. Am. Ceram. Soc., 8, 339-355; 789-794.

15. Tammann, G. and Hesse, W. (1926) Die Abhängigkeit der Viskosität von der Temperatur bei unterkühlten Flüssigkeiten. Z. Anorg. Allg. Chem., 156, 245-257.

16. Littleton, J.T. and Roberts, E.H. (1920) A method for determining the annealing temperature of glass. J. Opt. Soc. Amer. 4, 224-229. 
17. Lillie, H.R. (1954) Re-evaluation of glass viscosity at annealing and strain point. J. Am. Ceram. Soc., 37, $111-117$.

18. Littleton, J.T. (1940) The softening point of glasses. J. Soc. Glass Technol., 24, 176-185.

19. Littleton, J.T. (1927) A method for measuring the softening temperature of glasses. J. Am. Ceram. Soc., 10, $259-263$.

20. Lillie, H.R. (1952) A method for measuring the flow point of glass. J. Am. Ceram. Soc., 35, 149-155.

21. Dietzel, A. and Brückner, R. (1957) Ein Fixpunkt der Zähigkeit im Verarbeitungsbereich der Gläser. Schnellbestimmung des Viskositäts-Temperatur-Verlaufes. Glastech. Ber, 30, 73-79.

22. Deeg, E. (1958) Zusammenhang zwischen Feinbau und mechanisch-akustischen Eigenschaften einfacher Silikatgläser. Glastech. Ber., 31, 1-9, 85-93.

23. Stong, G.E. (1937) The modulus of elasticity of glasses. J. Am. Ceram. Soc., 20, 16-22.

24. Polanyi, M. (1921) Die Natur des Zerreissvorganges. Z. Phys., 7, 323-327.

25. Orowan, E. (1944) Fatigue of glass under stress. Nature, 154, 341-343.

26. Griffith, A.A. (1920) The phenomena of rupture and flow in solids. Phil. Trans. Roy. Soc. London, A221, $163-198$.

27. Inglis, E. (1913) Stresses in a plate due to pressure of cracks and sharp corners. Trans. Inst. Naval Arch., 55, 219.

28. Lawn, B.R. and Wilshaw, T.R. (1975) Fracture of Brittle Solids, Cambridge Solid State Science Series, Cambridge University Press, Cambridge

29. El-Shamy, T.M., Lewins, J. and Douglas, R.W. (1972) The dependence on the pH of the decomposition of glasses by aqueous solutions. Glass Technol., 13, 81-87.

30. Rana, M.A. and Douglas, R.W. (1961) The reaction between glass and water. Part 2. Discussion of the results. Phys. Chem. Glasses, 2, 196-205.

31. Scholze, H., Helmreich, D. and Bakardiev, I. (1975) Untersuchungen über das Verhalten von Kalk-Natrongläsern in verdünnten Säuren. Glastech. Ber., 48, 237-247. 